\title{
Mechanical properties of loosing natural fiber reinforced polypropylene
}

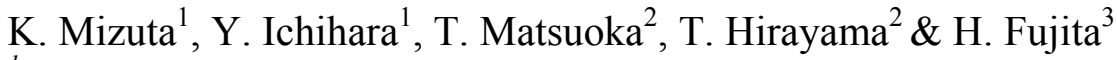 \\ ${ }^{1}$ Graduate Student, Doshisha University, Japan \\ ${ }^{2}$ Department of Mechanical Engineering, Doshisha University, Japan \\ ${ }^{3}$ Hyogo Prefectural Institute of Technology, Japan
}

\begin{abstract}
As interest in studying environmental issues has grown, the mechanical properties of eco-friendly materials have been studied. In our research, a card machine that enables raw materials to loose and to mix uniformly was used. Fibrous polypropylene and cotton or ramie fiber were used as raw materials since many textiles and apparel are made of such fibers. From a viewpoint of reuse, the loosing technique of the card machine can be effective. We designed anisotropic composites by using the card machine followed by the hot press process. The tensile, flexural and compression properties were investigated. Ramie fiber reinforced polypropylene (RP) was stronger than cotton fiber reinforced polypropylene (CP); however, breaking elongation of composites of $\mathrm{CP}$ is superior to that of RP because of the character of natural fiber. The density of composites applied to the loosing technique also affected the strength of composites. It was clear that density is an important parameter to describe the performance of a green composite. Moreover, it was found that the initial fracture in flexural behavior is affected by the compression strength of composites using the loosing technique.

Keywords: cotton fiber, Ramie fiber, loosing technique, tensile property, flexural property, compression property, density.
\end{abstract}

\section{Introduction}

The increasing concerns for environmental issues and the growing eco-friendly society direct universities and makers to development of 'Green composites' [1-4]. Green composites should be friendly for both environment and human 
body. Materials that can cause pollution and environmental load are not real green composites. Properties of various green composites have been studied; however, there are few reports about relationship between density and mechanical properties of composites. In our research, composites were made by using the loosing technique that enables raw materials to loose, to disperse and to mix uniformly. Natural fibers vary in their geometrical and mechanical properties. Therefore, it is important to grasp the interrelation between density and mechanical properties of composites. Tensile, flexural, and compression tests on cotton and ramie fiber reinforced polypropylene were performed to investigate these correlations.

\section{Experiment}

\subsection{Material}

Polypropylene (PP), a matrix resin, is a fibrous resin with a melting point of $165^{\circ} \mathrm{C}$. Cotton and ramie fibers were used as reinforcement. Length of cotton fiber was $30 \mathrm{~mm}$, and ramie fiber was $80 \mathrm{~mm}$.

\subsection{Production of composite}

\subsubsection{Loosing method}

Loosing of fibrous PP resin and natural fiber was performed by a card machine. In mixing of the fibrous resin and the natural fiber, it is important to distribute the fiber uniformly in resin. Therefore, a loosing fibrous resin sheet and a loosing fiber sheet were separately made by the card machine before mixing fibrous rein and fiber.

\subsubsection{Molding method}

Specimens were manufactured by a vacuum compressed molding method as shown in Fig.1. In this research, molding conditions were selected as shown in Table 1 .

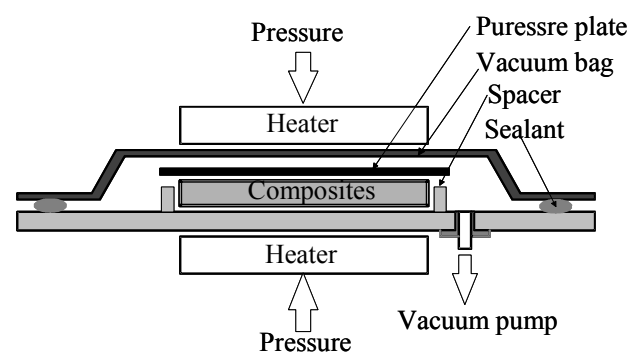

Figure 1: Vacuum compressed molding method. 
Table 1: $\quad$ Molding condition.

\begin{tabular}{c|c|c|c}
\hline \hline Weight fraction of fiber [\%] & 0 & 30 & 50 \\
\hline Molding pressure [MPa] & 1 & 10 & 15 \\
\hline Molding temperature $\left[{ }^{\circ} \mathrm{C}\right]$ & \multicolumn{3}{|c}{210} \\
\hline Molding time $[\mathrm{min}]$ & \multicolumn{3}{|c}{10} \\
\hline
\end{tabular}

\subsection{Experimental method}

\subsubsection{Static tensile test}

The test pieces were cut to a rectangle $(20 \times 130 \times 2 \mathrm{~mm})$ from a plate, and gage length was $80 \mathrm{~mm}$. The static tensile tests were carried out at the test speed 1.0 $\mathrm{mm} / \mathrm{min}$ under a room temperature by an INSTRON testing machine (Type 4467).

\subsubsection{Flexural test}

Dimensions of specimen were $15 \times 50 \times 2 \mathrm{~mm}$. A three points flexural test was carried out at the test speed $1.0 \mathrm{~mm} / \mathrm{min}$ under a room temperature by an INSTRON testing machine (Type 4467). Span length was $30 \mathrm{~mm}$.

\subsubsection{Compression test}

Dimension of specimens were $3 \times 2 \times 55 \mathrm{~mm}$, and gage length was $17 \mathrm{~mm}$. The compression tests were carried out at the test speed $1.0 \mathrm{~mm} / \mathrm{min}$ under a room temperature by a testing machine (Ez Test-500, SHIMADZU. Corp.). Specimen holding assembly was used to avoid a buckling.

\section{Results and discussion}

\subsection{Tensile test}

\subsubsection{Effect of loosing technique}

Fig. 2 shows typical S-S curves of cotton fiber reinforced PP (CP) and ramie fiber reinforced PP (RP) in loosing direction. The number following $\mathrm{CP}$ or RP means fiber contents. RP is stronger than $\mathrm{CP}$ in any fiber contents, while deformation ability of $\mathrm{CP}$ is superior to that of RP by more than $50 \%$. It was caused by properties of natural fiber; cotton fiber has many crimps and twists complexly each other, while ramie fiber is relatively high strength and modulus. Fig.3 shows the effect of the loosing technique, where $\theta$ represents the angle between loosing direction and tensile direction. The loosing technique could order fiber orientation in both CP and RP, and anisotropic composites could be obtained.

\subsubsection{Effect of fiber length on loosing process}

Strong orientation couldn't be seen in short ramie (SRP: fiber length=30 mm) in Fig.3. This result indicates the difference of the effect of fiber length on loosing 
degree. It was clear that to order orientation of short ramie fibers was more difficult than long fibers by measuring fiber orientation angle from flatwise direction of specimen. It was one of the reasons that made SRP weak compared to RP.

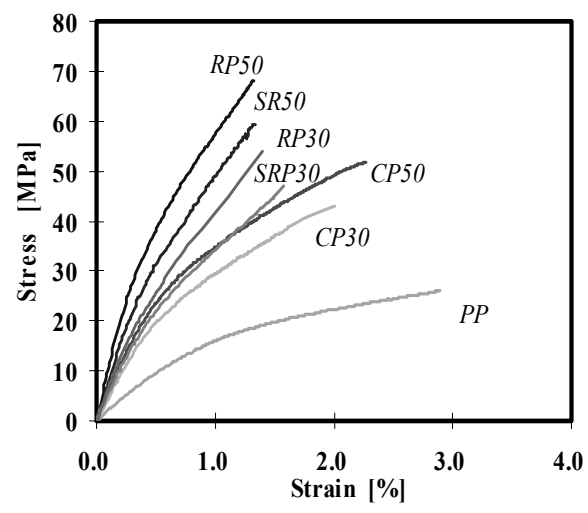

Figure 2: $\quad$ S-S curves of composites.

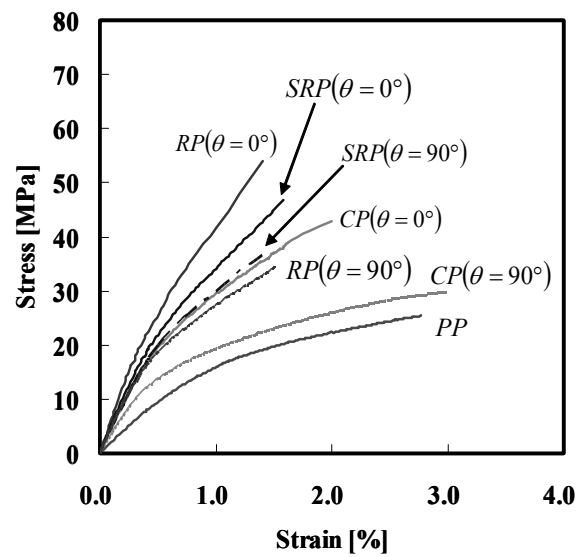

Figure 3: Anisotropy of loosing natural fiber reinforced polypropylene (Fiber contents: $30 \mathrm{wt} . \%)$.

\subsection{Flexural test}

Fig. 4 shows flexural behavior of composites. Table 2 shows flexural strength and flexural modulus of composites. Flexural modulus was improved in all materials, while, flexural strength was not increased well on the whole. However, density is an important parameter to estimate the loosing natural fiber reinforced 
polypropylene. The relationship between density and strength properties is discussed in the next section.

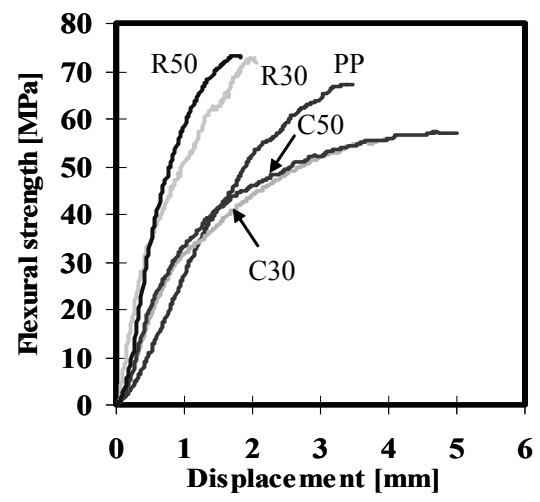

Figure 4: $\quad$ Flexural behavior of NFRP.

Table 2: $\quad$ Flexural strength and modulus of NFRP.

\begin{tabular}{l|c|c|c|c|c}
\hline & PP & C30 & C50 & R30 & R50 \\
\hline Flexural strength [MPa] & 65 & 55 & 64 & 69 & 66 \\
\hline Flexural modulus [GPa] & 1.9 & 2.2 & 2.7 & 4.4 & 3.8 \\
\hline
\end{tabular}

\subsection{Effect of density on mechanical properties}

\subsubsection{Correlation between density and flexural strength}

Fig.5 shows the effect of density on flexural strength of CP and RP. Composites using loosing technique have low density because the loosing sheet that consists of fibrous PP and natural fiber contains a lot of voids. However, there is a specific positive correlation between them. Strength of composites, which had densities from 0.75 to $0.85 \mathrm{~g} / \mathrm{cm}^{3}$, was almost same to that of PP.

That means specific strength increased by around $20 \%$ since the density of PP is $0.91 \mathrm{~g} / \mathrm{cm}^{3}$. It was also suggested that high density composites yield high strength. Therefore, high density composites were manufactured applying higher pressure. In this paper, we classified into low density materials (LD) and high density materials (HD). As a result, composites with around $1.0 \mathrm{~g} / \mathrm{cm}^{3}$ of density were obtained. Flexural strength of high density composites HD C30 and HD R50 could be improved except for HD R30 as shown in Fig.5.

\subsubsection{Correlation between density and tensile strength}

Static tensile tests were also carried out to investigate tensile behavior of high density composites. As a result, tensile strength of high density composites decreased (Fig.6). In this decrease effect, the higher the natural fiber content was, the lower the tensile strength of the composites was. This indicates the effect of molding pressure on natural fiber. In the high pressure molding process, massive 
damage of natural fibers could be the cause of this result. Observation of natural fibers that were exposed to fracture surfaces is shown in the next section.

The relationship between some parameters such as strength, crystallization degree and density of PP resin is nearly linear [5]. It is thought that the difference of crystallization degree between low density composites and high density composites is also one of the reasons for these results.

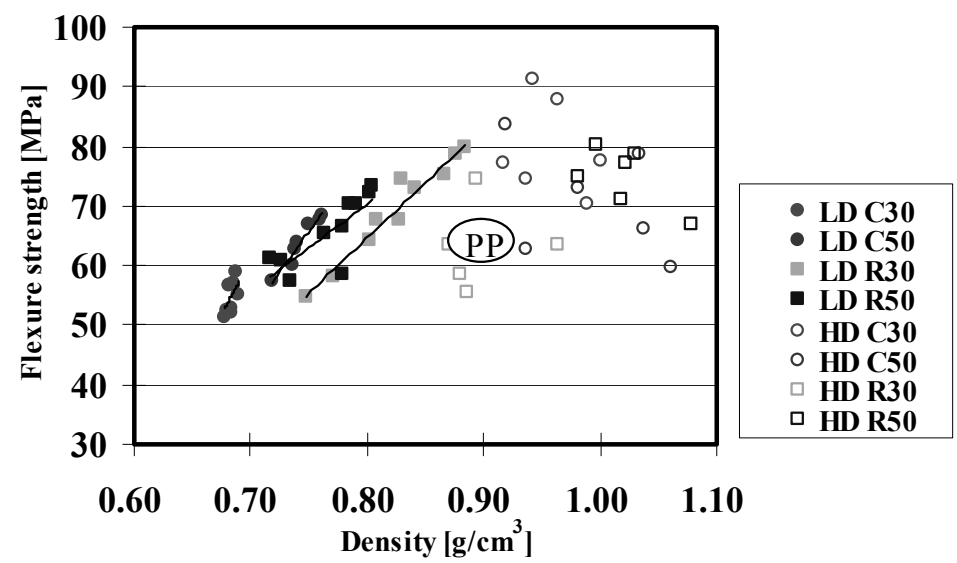

Figure 5: Correlation between density and flexural strength.

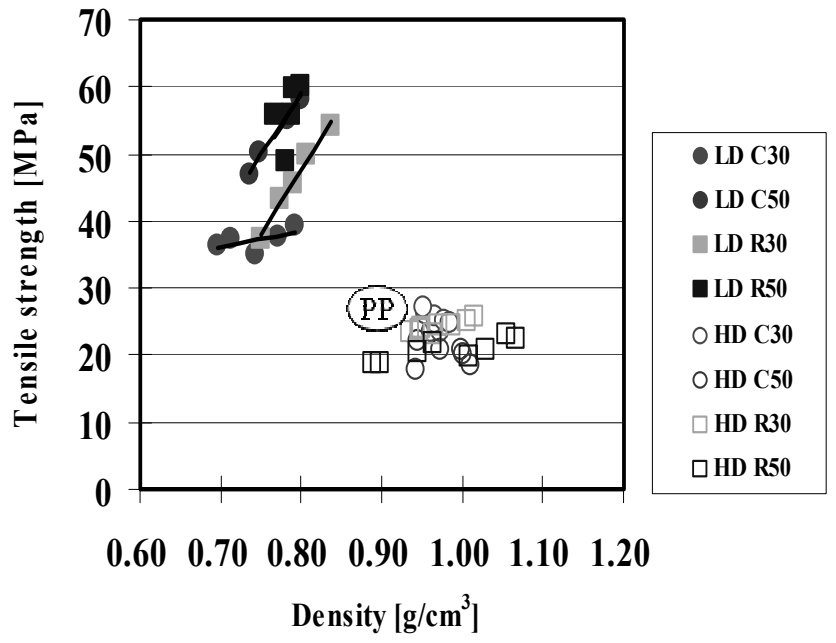

Figure 6: Correlation between density and tensile strength. 


\subsection{Difference of fracture surface between low $\&$ high density material}

Fig.7 shows SEM photograph of tensile fracture surface of low density and high density CP, respectively. There are many pull out single fibers in both, however these forms are different. A notable point is the difference among their lengths. In the high density composite, fibers were evenly broken shortly. Therefore, it showed relatively flat fracture surface. It was also observed in the fracture surface of CP.

The form of the tensile fracture surface is determined by the relative degree and friction force between fiber and resin. If the ratio of tensile strength to interfacial shear strength is relatively small, then the fracture surface is flat [6]. In this research, high pressure was applied to manufacture high density composites. Fatal damage caused by high molding pressure decreased the potential of reinforcement by the natural fiber. Therefore, it is thought the massive damage on the crossing point of fibers made high density composites low strength.

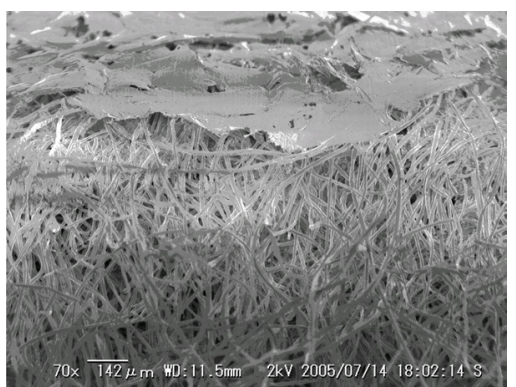

(a) Low density CP $\quad 142 \mu \mathrm{m}$

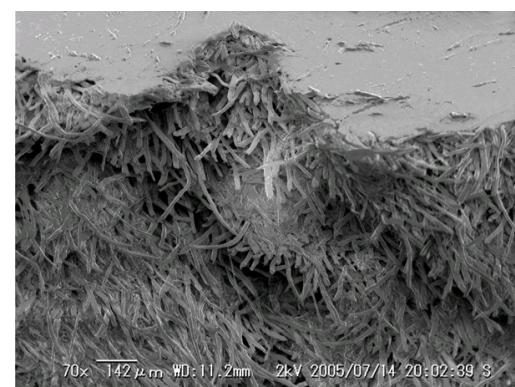

(b) High density CP $\quad 142 \mu \mathrm{m}$

Figure 7: Fracture surface of CP (Fiber contents: 50wt.\%).

\subsection{Compression test}

Strengthening was not found in flexural strength in spite of the improvement of tensile strength. Therefore it is thought that compression property weakens the flexural strength. To investigate the cause of this degradation, compression test was carried out. Fig. 8 shows compression strength of composites. As a result, compression strength decreased by $30-50 \%$ compared to polypropylene, however, high density materials were superior to low density materials only in compression strength. It was identified compression failure causes in flexural behavior. More detail of relationship between compression strength and flexural behavior is discussed in the next section.

\subsection{Effect of compression strength on flexural behavior}

According to Bazhenov [7], flexural stress-displacement curve can be divided three parts as Fig.9. The transition point from region I to region II is called Knee 
point. At Knee point, composite reaches compression yielding point, and then relationship between flexural stress and displacement starts non liner behavior. Fig.9 shows flexural stress at Knee point in flexural behavior.

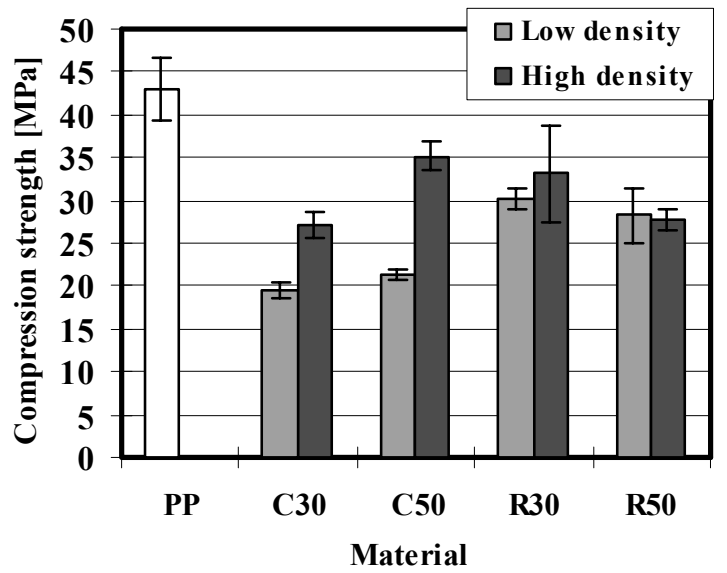

Figure 8: $\quad$ Effect of density on compression strength.

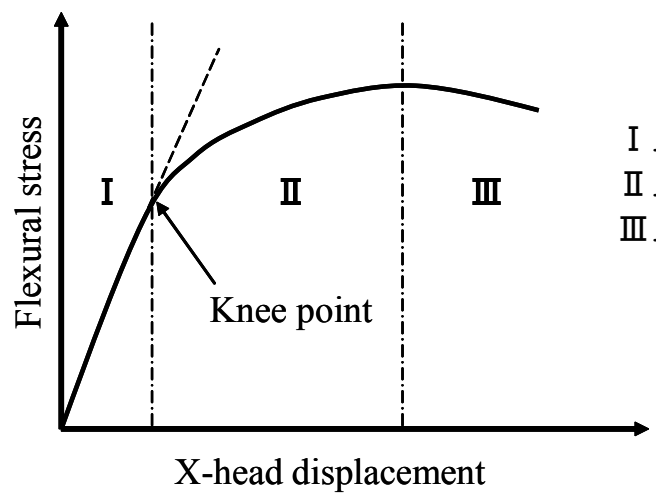

Figure 9: $\quad$ Schematic illustrating three stages in flexural deformation.

Compared Fig.8 with Fig.10, we confirmed correlationship between flexural stress at Knee point and compression strength. Compression stress was nearly to the flexural stress at Knee point about both CP and RP. Furthermore, high density materials tend to be superior to low density materials in Fig.8. Reflecting such a tendency, Knee point stress of high density materials is superior to that of low density materials (Fig.10). Therefore, it is necessary to keep compression strength as much as possible to improve flexural strength of loosing natural fiber reinforced polypropylene. 


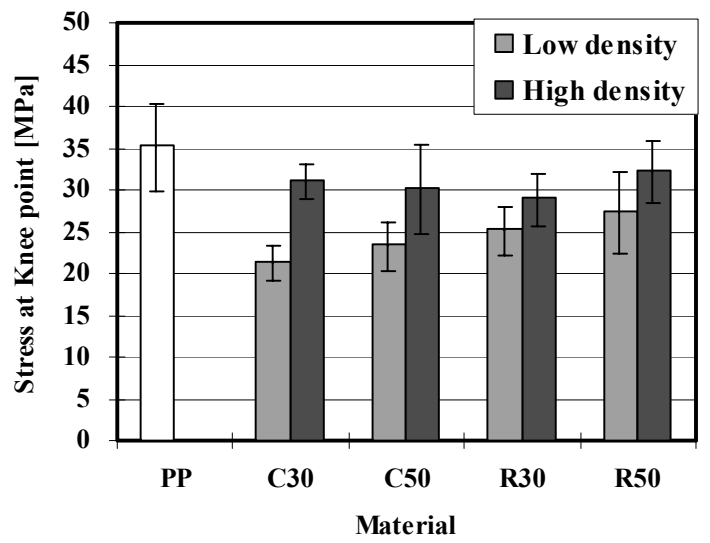

Figure 10: Stress at knee point in flexural test.

\section{Conclusion}

Cotton fiber and ramie fiber reinforced polypropylene were manufactured using loosing technique. The experimental data showed that:

(1) Loosing technique enables to uniform fiber orientation in both cotton fiber reinforced polypropylene and ramie fiber reinforced polypropylene.

(2) Long fibers (fiber length $=80 \mathrm{~mm}$ ) were easier than short fiber (fiber length $=30 \mathrm{~mm}$ ) to orient natural fiber uniformly in ramie fiber reinforced polypropylene.

(3) There was a positive correlationship between mechanical properties such as tensile strength and flexural strength and density in low density materials.

(4) Damage of natural fiber by high pressure caused weakness of tensile strength of composites.

(5) Initial fracture in flexural behavior of loosing natural fiber reinforced polypropylene was affected by compression strength. Therefore, improvement of compression strength is necessary to obtain higher performance of loosing natural fiber reinforced polypropylene.

\section{Acknowledgements}

Authors would like to express special thanks to CHISSO Ltd. for providing fibrous PP resin in this study. This study was supported by the Academic Frontier Research Project on "New Frontier of Biomedical Engineering Research" of ministry of education, culture, sports, science, and technology.

\section{References}

[1] Kenji Ohnishi, Kazunori Umeoka, Hideyuki Ando, and Wenhai Liu. Matsushita Electric Works, Ltd., Technical Report, pp58-63, 2003. 
198 High Performance Structures and Materials III

[2] Teruo Kimura, J. Soc. Mat. Sci, Japan, Vol.50, No10, pp.1158-1163, 2001.

[3] Hiroshi Yamamoto, Takashi Matsuoka, Kazuhiko Sakaguchi, and Hiroyuki Fujita, Proceedings of Third International Workshop on Green Composites, pp.95-100, 2005.

[4] Anil N Netravali, Proceedings of Third International Workshop on Green Composites, pp.11-15, 2005.

[5] Kaneyuki Takagi, Heizo Sasaki, Polypropylene resin, Nikkan Kogyo, Tokyo (1970).

[6] D. Hall, An Introduction to Composite Materials, Baifukan Ltd., Tokyo (1984).

[7] S.L. Bazhenov, Composites 26, pp.757-765, 1995. 